\title{
Internet-based survey of the frequency and types of orthopedic conditions and injuries experienced by dogs competing in agility
}

Arielle Pechette Markley DVM

Abigail B. Shoben $\mathrm{PhD}$

Nina R. Kieves DVM

From the Veterinary Medical Center (Pechette Markley) and Department of Clinical Sciences (Kieves), College of Veterinary Medicine, and Division of Biostatistics, College of Public Health (Shoben), The Ohio State University, Columbus, Ohio.

Address correspondence to Dr. Pechette Markley (markley.125@osu.edu).

\begin{abstract}
OBJECTIVE
To describe the frequency and types of injuries experienced by dogs competing and training in agility and identify breed and geographic differences in frequency and types of injuries.
\end{abstract}

\section{SAMPLE}

Surveys completed by owners of 4,70 I dogs.

\section{PROCEDURES}

The study involved an internet-based survey. Participants were asked whether their dog had ever had an injury that kept it from participating in agility for > I week and, if so, to identify the location and type of injury.

\begin{abstract}
RESULTS
Owners of I,958 (4I.7\%) dogs reported that their dogs had experienced an injury. The most common injury locations were the shoulder region $(\mathrm{n}=$ 589 [30.1\% of all dogs with an injury]) and iliopsoas muscle (380 [19.4\%]). The percentage of Border Collies sustaining an injury (549/I,052 [5I.9\%]) was significantly higher than percentages of other breeds. Percentage of dogs that sustained an injury varied by country, with the highest percentage reported in Australia (93/174 [53.4\%]) and lowest percentage reported in the US (I,I49/2,889 [39.8\%]).

\section{CONCLUSIONS AND CLINICAL RELEVANCE}

Results suggested that, among dogs competing and training in agility, injuries to the shoulder region were substantially more common than injuries in other anatomic locations, with iliopsoas muscle injuries second most common. The frequency and types of injuries varied among breeds and geographic regions. Findings may help guide clinical evaluations when agility dogs are seen in clinical practice for performance issues or lameness. Further studies regarding regional differences in injury rates are required.
\end{abstract}

A gility is one of the most popular canine performance events in the world, with participation continuing to grow rapidly. For agility events licensed by the American Kennel Club, for example, the total number of entries increased $38 \%$ over the past decade from 870,603 entries in 2009 to 1,202,711 in 2019 (C DeYoung, Director of Agility, American Kennel Club, email, June 30, 2020). Previous surveys ${ }^{1-3}$ evaluated the prevalence of injuries among dogs training and competing in agility and found that $32 \%$ of dogs had experienced an injury, with injuries of the shoulder region (22.9\%), back (18.5\%), digits (13.3\%), and neck (12.4\%) most commonly reported. However, these surveys were conducted $>10$ years ago, and to our knowledge, there have been no updated evaluations of the prevalence and types of injuries sustained by agility dogs even though the sport has changed dramatically in the past 10 years.

As the sport has grown, the level of competition has increased, courses have become more challenging, and approaches to certain agility obstacles have become more extreme. ${ }^{4,5}$ These changes place increased physical demands on participating dogs and may potentially increase the risk of injury. On the other hand, changes have been made over the past 10 years to improve the safety of agility equipment and some agility obstacles, such as the chute, have been removed from competition entirely by certain organizations. ${ }^{6-8}$

Course and obstacle requirements vary between sponsoring organizations both within the US and between the US and other countries., ${ }^{5-19}$ Spacing between obstacles, jump heights, obstacle dimensions, and numbers of required obstacles are all regulated by the sponsoring organization and can therefore vary widely both within and between geographic regions. There are also considerable differences in the sequencing of obstacles depending on the level of competition, the judge designing the course, and the event venue size and layout. These variations, along with regional differences in handling and training techniques, can potentially alter the physical demands on competing dogs.

Further, even as courses have become more technically demanding, the speed at which dogs complete those courses, at least at large international events, has increased. ${ }^{20,21}$ At the same time, the percentage of dogs successfully completing these courses without any faults or mistakes has decreased, reflecting greater technical challenges. ${ }^{20,21}$ 
As agility has increased in popularity, more owners are acquiring dogs for the specific purpose of competing in agility and more breeders are specializing in producing dogs to specifically meet the demands of agility competition. Dogs of breeds noted for fast speeds, high drive, and high trainability are sought out. Given that breeds differ in temperament, running style, and conformation, it is likely that different breeds are more or less prone to injuries or to different types of injuries, as is the case for equine athletes. ${ }^{22,23}$

Over the past decade, there have been substantial advancements in the field of veterinary medicine, particularly with regard to sports medicine and rehabilitation. Our understanding of musculoskeletal injuries has increased, and advanced diagnostic modalities, such as musculoskeletal ultrasonography, arthroscopy, and MRI, have become more widely available. In addition, with the development of online learning platforms and social media, there has been an increase in awareness of injuries and in familiarity with specific types of injuries among both veterinary professionals and dog owners.

For all of these reasons, updated information on injuries among dogs competing and training in agility is needed. The purposes of the study reported here were to describe the frequency and types of injuries experienced by dogs competing and training in agility and identify breed and geographic differences in frequency and types of injuries. On the basis of the authors' clinical experience and caseload, we hypothesized that the prevalence of injuries would be higher than it was 10 years ago and that injuries to the shoulder region and iliopsoas muscle would be most commonly reported. We also hypothesized that there would be breed and regional differences with regard to frequency and types of injuries.

\section{Materials and Methods}

An internet-based survey (online survey software; Qualtrics; copies of the questionnaire are available from the corresponding author on request) conducted in English was distributed primarily via social media (Facebook Inc) during a 6-week period in the fall of 2019. Individuals were eligible to participate if they owned at least 1 dog that had competed in agility in the previous 3 years. Participants who owned $>1$ eligible dog were instructed to fill out the survey first for the dog whose name was closest to the letter A to facilitate random selection of the initial dog. Participants could then complete the survey for additional dogs. The survey and research protocol were reviewed and approved by the institutional review board at The Ohio State University.

For injury data, participants were asked whether their dog had ever had an injury that kept it from participating in agility for $>1$ week. If yes, they were asked to identify all locations on the body where their dog had been injured (Figure I). Participants were then asked to respond to additional questions based on the body parts selected. For each body part selected, participants were asked to denote whether the dog had ever had any of a list of injuries specific to that body part, with an option to add additional text if needed. During tabulation of the surveys, this text was reviewed and the type of injury was classified as a known specific injury or was designated as unknown (ie, unspecified, nonspecific, or unknown injury).

We defined geographic regions on the basis of geographic proximity and similarity of the rules and regulations for the various organizations that licensed or sanctioned agility trials. Final geographic regions were the US, Canada, Australia, New Zealand, Scandinavia, Continental Europe, the UK and Ireland, and all other countries.

Descriptive statistics were calculated for the frequency and types of injuries. Associations between injury history (yes vs no) and breed or geographic region were assessed with the $\chi^{2}$ or Fisher exact test. To avoid small cell sizes, individual breeds and geographic regions were included in assessments of injury history if there were $\geq 50$ dogs in the sample of that breed or from that region. Individual breeds and regions were included in the comparison of injury locations if there were $\geq 85$ dogs reporting an injury. All other breeds and regions were collapsed into an "other" category. Because the comparisons between types of injuries by breed and region were secondary analyses, we considered values of $P<0.05$ to be significant; however, we also noted which comparisons remained significant after use of the Holm correction for multiple comparisons within analyses by breed and region. Finally, to account for potential breed differences between regions, we included both breed and region as covariates in a logistic regression model with injury history as the outcome variable.

\section{Results}

The survey was started by 5,113 individuals and completed (defined as $>90 \%$ survey completion and answering the primary injury history question) by

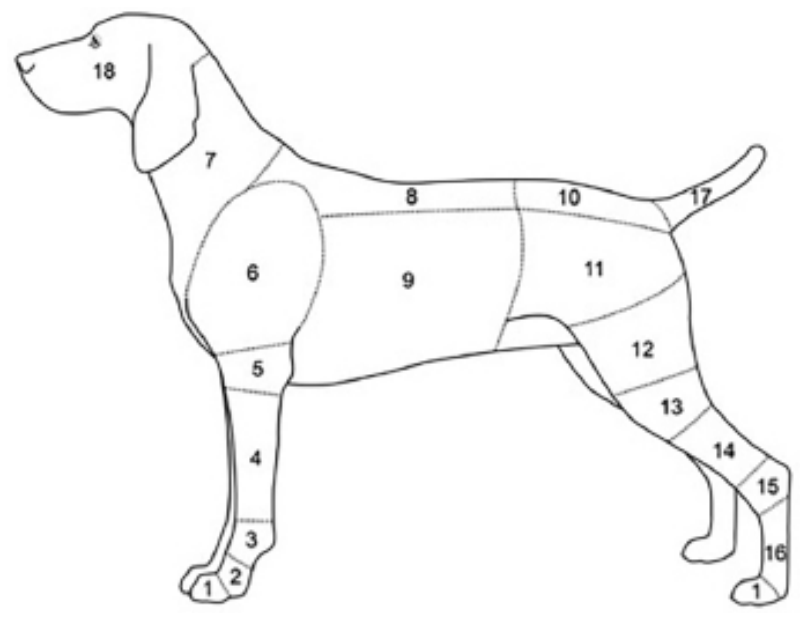

Figure I-Diagram illustrating anatomic regions used in a survey of the frequency and types of injuries experienced by dogs competing and training in agility. Note that region I was divided into 2 parts (digits [a] and paw pads [b]) and that region II was also divided into 2 parts (hip region [a] and iliopsoas muscle [b]). 
4,197 individuals. A small portion of these individuals voluntarily provided data on additional dogs, and thus, the total number of dogs included was 4,701 . For those individuals who completed the survey, median response time was 18 minutes (interquartile [25th to 75th percentile] range, 15 to 25 minutes).

Mean \pm SD age of the dogs at the time of the survey was $6.3 \pm 2.6$ years; most $(4,417 / 4,665$ [95\%]) dogs were $\leq 11$ years old. Of the 4,693 dogs for which information was available, most $(n=3,448[73.5 \%])$ had been acquired from a breeder, with 800 (17.0\%) having been acquired from a rescue organization or shelter and 445 (9.5\%) having been acquired by some other means. Most $(3,375 / 4,693$ [71.9\%]) of these dogs had been acquired with agility in mind. Age at the time the dog was acquired was < 8 weeks for 981 (20.9\%) dogs, 8 to 12 weeks for 2,517 (53.6\%), 13 to 15 weeks for 248 (5.3\%), 4 to 6 months for 278 (5.9\%), 7 to 12 months for 264 (5.6\%), and $>12$ months for 405 (8.6\%). Of the 4,701 dogs, 549 (11.7\%) were sexually intact females, 1,854 (39.4\%) were spayed females, 768 (16.3\%) were sexually intact males, and 1,530 (32.5\%) were neutered males. For 3,390 (72.1\%) of 4,701 dogs, agility was the dog's main sport focus; for 916 (19.5\%), agility was mostly the main sport focus; and for 394 (8.4\%), agility was not the main sport focus.

The most common breed or type in our sample was Border Collie, followed by mixed-breed, Shetland Sheepdog, and Australian Shepherd (Table I). The most common geographic region was the US, followed by the UK and Ireland, Canada, and Continental Europe (Table 2).

Overall, owners of 1,958 of the 4,701 (41.7\%) dogs reported that the dog had experienced an injury severe enough to keep it from participating in agility for $\geq 1$ week. Owners reported that of the 1958 dogs with this information available, 986 (50.6\%) had experienced only 1 such injury, 569 (29.2\%) had experienced 2 such injuries, 233 (11.9\%) had experienced 3 such injuries, 72 (3.7\%) had experienced 4 such injuries, 23 (1.2\%) had experienced 5 such injuries, and 67 (3.4\%) had experienced $\geq 6$ such injuries.

The percentage of dogs that had sustained at least 1 injury varied significantly by breed $(P<0.001$; Table $1)$ and geographic region $(P=0.003$; Table 2$)$. The overall significant variation by breed was attributable to the high rate observed among Border Collies (546/1,052 [51.9\%]) and the low rate observed among Poodles (21/91 [23.1\%]), as determined on the basis of adjusted Pearson residuals of 7.6 and 3.6, respectively. The overall significant variation by geographic region was attributable to the high rate observed in Australia (93/174 [53.4\%]) and the low rate observed in the US $(1,149 / 2,889$ [39.8\%]), with adjusted Pearson residuals of 3.2 and 3.4, respectively. Differences in injury history by breed and country persisted even when adjusting for each other in a logistic regression model $(P<0.001$ and $P=0.012$, respectively).

Among injured dogs, the most common injury location was the shoulder region (589/1,958 [30.1\%] injured dogs), followed by the iliopsoas muscle, digits, and lumbar spinal or lumbosacral region (Table 3). Among the 589
Table I-Distribution of breed and type for dogs $(n=4,675)$ whose owners responded to a survey concerning the frequency and types of injuries (ie, an injury severe enough to keep the dog from participating in agility for $\geq$ I week) experienced by dogs competing and training in agility.

No. $(\% \dagger)$ of dogs

Breed or type No. (\%*) of dogs with at least I injury

Australian Shepherd

Border Collie

Cocker Spaniel

German Shepherd Dog

Golden Retriever

Labrador Retriever

Mixed breed

Other

Papillon

Pembroke Welsh Corgi

Poodle

Shetland Sheepdog

$312(6.7)$

I,052 (22.5)

$82(1.8)$

$57(1.2)$

146 (3.I)

$161(3.4)$

$616(13.2)$

$1,656(35.4)$

96 (2.I)

$90(1.9)$

$91(2.0)$

$316(6.8)$

$121(38.8)$

$546(51.9)$

30 (36.6)

24 (42.I)

$51(34.9)$

$60(37.3)$

$240(39.0)$

$653(39.4)$

$36(37.5)$

$44(48.9)$

$21(23.1)$

$125(39.6)$

A total of 4,197 owners responded to the survey and provided information on 4,70 I dogs; not all owners answered all questions.

*Calculated as percentage of dogs in the overall sample. †Calculated as percentage of dogs in that row with at least I injury.

Table 2-Geographic distribution of the dogs $(n=4,648)$ in Table I.

\begin{tabular}{lcc}
$\begin{array}{l}\text { Country or } \\
\text { geographic region }\end{array}$ & No. (\%*) of dogs & $\begin{array}{c}\text { No. (\%†) of dogs } \\
\text { with at least I injury }\end{array}$ \\
\hline Australia & $174(3.7)$ & $93(53.5)$ \\
Canada & $432(9.3)$ & $178(41.2)$ \\
Continental Europe & $365(7.9)$ & $168(46.0)$ \\
New Zealand & $109(2.4)$ & $50(45.9)$ \\
Other & $51(1.1)$ & $21(41.2)$ \\
Scandinavia & $132(2.8)$ & $65(49.2)$ \\
UK and Ireland & $496(10.7)$ & $215(43.4)$ \\
US & $2,889(62.2)$ & $1,149(39.8)$
\end{tabular}

Table 3-Anatomic distribution of injuries experienced by the dogs in Table 1.

\begin{tabular}{lcc} 
Anatomic region & No. (\%) of dogs & $\begin{array}{c}\text { Percentage of } \\
\text { injuries }\end{array}$ \\
\hline All regions & $1,958(41.7)$ & 100 \\
Shoulder & $589(12.5)$ & 30.1 \\
Iliopsoas muscle & $380(8.1)$ & 19.4 \\
Digits (toes) & $350(7.4)$ & 17.9 \\
Lumbar spinal or lumbosacral & $336(7.1)$ & 17.2 \\
Stifle & $261(5.6)$ & 13.3 \\
Thoracic spinal & $258(5.5)$ & 13.2 \\
Paw pads & $200(4.3)$ & 10.2 \\
Neck & $199(4.2)$ & 10.2 \\
Hip & $169(3.6)$ & 8.6 \\
Carpal & $166(3.5)$ & 8.5 \\
Thigh & $116(2.5)$ & 5.9 \\
Elbow & $90(1.9)$ & 4.6 \\
Metacarpal & $63(1.3)$ & 3.2 \\
Ribs or side & $55(1.2)$ & 2.8 \\
Antebrachium & $41(0.9)$ & 2.1 \\
Head & $39(0.8)$ & 2.0 \\
Tibial or fibular & $34(0.7)$ & 1.7 \\
Tarsal or calcaneal tendon & $34(0.7)$ & 1.7 \\
Tail & $24(0.5)$ & 1.2 \\
Metatarsal & $23(0.5)$ & 1.2 \\
\hline
\end{tabular}

Some dogs experienced > I injury. 
dogs with shoulder injuries, the most common injury was biceps tendinopathy $(n=113)$, followed by medial shoulder syndrome or instability (75), and supraspinatus tendinopathy (62; Table 4). Similarly, among the 380 dogs with iliopsoas muscle injuries, the most common injury was muscle strain $(n=328)$. Of the 350 dogs with digit injuries, the most commonly reported injuries were sprains and strains ( $n=137)$, torn nails (123), and fractures (47). The most common injuries in the 336 dogs with lumbosacral disease were intervertebral disk disease $(n=42)$, lumbosacral instability (26), and lumbosacral stenosis (25).

For dogs that had sustained at least 1 injury, the location of injury varied significantly by breed (Table 5 ). Iliopsoas muscle injuries were significantly $(P=0.002)$ more common among Border Collies, Shetland Sheepdogs, and Australian
Shepherds and less common among mixed-breed dogs and dogs of other breeds. Injuries of the thoracic spinal region were significantly $(P<0.001)$ more common among Shetland Sheepdogs, Australian Shepherds, and dogs of other breeds and less common among Border Collies and mixedbreed dogs. Injuries of the neck region were significantly $(P$ $<0.001)$ more common among Shetland Sheepdogs and less common among Border Collies. For these 3 locations, significant variations among breeds were still identified after use of the Holm correction for multiple comparisons. For other locations, including the digits, paw pads, and tail, differences among breeds were significant at values of $P<0.05$.

In addition, injury location for dogs that had sustained at least 1 injury varied significantly by geographic region (Table 6). Iliopsoas muscle injuries were

Table 4-Most commonly reported injuries and orthopedic conditions, by anatomic region, for the dogs in Table I.

Injury or condition (No. of dogs)

\begin{tabular}{|c|c|c|c|}
\hline Anatomic region & Most common & Second most common & Third most common \\
\hline Shoulder & Biceps tendinopathy $(1 \mid 3)$ & Medial shoulder syndrome or instability (75) & Supraspinatus tendinopathy $(62)$ \\
\hline Iliopsoas muscle & Strain $(328)$ & Avulsion or tear (32) & Contusion; dog bite (tie; I each) \\
\hline Digits (toes) & Sprain or strain (137) & Torn nail (123) & Fracture (47) \\
\hline Lumbar spinal or lumbosacral & Intervertebral disk disease (42) & Lumbosacral instability (26) & Lumbosacral stenosis (25) \\
\hline Stifle & Cranial cruciate ligament rupture or tear (128) & Medial patella luxation (29) & Arthritis (17) \\
\hline Thoracic spinal & Intervertebral disk disease (40) & Arthritis (27) & Strain (23) \\
\hline Paw pads & Laceration (99) & Abrasion (90) & Burn (25) \\
\hline Neck & Nerve impingement (32) & Arthritis (16) & Intervertebral disk disease (16) \\
\hline Carpal & Strain (66) & Arthritis (30) & Hyperextension injury (I7) \\
\hline Thigh & Quadriceps muscle strain or tear (32) & Hamstring muscle strain or tear (29) & Gracilis muscle strain or tear (15) \\
\hline Elbow & Arthritis (15) & Sprain or strain (14) & Elbow dysplasia (13) \\
\hline Metacarpal & Sprain or strain (14) & Laceration (10) & Abrasion (5) \\
\hline Ribs or side & Laceration (II) & Contusion (6) & Bite wound (3) \\
\hline Antebrachium & Sprain or strain (9) & Fracture (7) & Laceration (7) \\
\hline Head & Tooth fracture (9) & Corneal ulcer (5) & Laceration; neoplasia (tie; 3 each) \\
\hline Tibial or fibular & Fracture (7) & Muscle strain or tear (5) & Laceration (3) \\
\hline Metatarsal & Abrasion (5) & Laceration (3) & Fracture (2) \\
\hline
\end{tabular}

Table 5-Percentages of dogs in Table I with an injury in various anatomic regions, by breed or type of dog.

\begin{tabular}{|c|c|c|c|c|c|c|}
\hline Anatomic region & $\begin{array}{l}\text { Border Collie } \\
\quad(n=546)\end{array}$ & $\begin{array}{c}\text { Mixed breed } \\
\quad(n=240)\end{array}$ & $\begin{array}{l}\text { Shetland } \\
\text { Sheepdog } \\
(n=125)\end{array}$ & $\begin{array}{l}\text { Australian } \\
\text { Shepherd } \\
(n=12 \mid)\end{array}$ & $\begin{array}{c}\text { Other } \\
\text { breed } \\
(n=926)\end{array}$ & $P$ value \\
\hline Shoulder & 30.0 & 26.3 & 34.4 & 28.9 & 30.7 & 0.56 \\
\hline Iliopsoas muscle & 23.4 & 13.8 & 23.2 & 24.8 & 17.3 & $0.002 *$ \\
\hline Digits (toes) & 21.2 & 18.3 & 11.2 & 20.7 & 16.3 & $0.037 \dagger$ \\
\hline Lumbar spinal or lumbosacral & 15.9 & 17.5 & 18.4 & 16.5 & 17.7 & 0.92 \\
\hline Stifle & 12.5 & 14.6 & 11.2 & 11.6 & 14.0 & 0.75 \\
\hline Paw pads & 12.6 & 12.5 & 4.8 & 14.0 & 8.4 & $0.007 \dagger$ \\
\hline Neck & 4.4 & 7.9 & 16.0 & 7.4 & 13.7 & $<0.001 *$ \\
\hline Hip & 7.9 & 8.8 & 7.2 & 9.9 & 9.1 & 0.89 \\
\hline Carpal & 9.5 & 8.8 & 8.8 & 12.4 & 7.2 & 0.28 \\
\hline Thigh & 7.7 & 5.0 & 8.0 & 4.1 & 5.1 & 0.18 \\
\hline Elbow & 4.6 & 5.4 & 7.2 & 4.1 & 4.1 & 0.54 \\
\hline Metacarpal & 3.3 & 2.9 & 4.8 & 3.3 & 3.0 & 0.84 \\
\hline Ribs or side & 2.0 & 4.6 & 3.2 & 3.3 & 2.7 & 0.32 \\
\hline Head & 1.3 & 3.3 & 4.0 & 0.8 & 1.9 & 0.13 \\
\hline Tibial or fibular & 2.9 & 2.5 & 0.8 & 1.7 & 1.0 & 0.063 \\
\hline Tarsal or calcaneal tendon & 2.2 & 1.7 & 2.4 & 0.8 & 1.5 & 0.76 \\
\hline Tail & 0.2 & 2.5 & 0.0 & 0.8 & 1.7 & $0.010+$ \\
\hline Metatarsal & 1.5 & 1.7 & 1.6 & 0.8 & 0.9 & 0.62 \\
\hline
\end{tabular}

Data represent percentage of dogs of each breed or type reported to have an injury in that anatomic region.

*Significant difference among breeds after Holm correction for multiple comparisons among the 20 anatomic regions. + Significant difference among breeds without Holm correction for multiple comparisons. 
Table 6-Percentages of dogs in Table I with an injury in various anatomic regions, by country or geographic region.

\begin{tabular}{|c|c|c|c|c|c|c|c|}
\hline Anatomic region & $\begin{array}{c}\text { US } \\
(n=I, I 49)\end{array}$ & $\begin{array}{c}\text { UK } \\
\text { and Ireland } \\
(n=215)\end{array}$ & $\begin{array}{l}\text { Canada } \\
(n=178)\end{array}$ & $\begin{array}{c}\text { Continental } \\
\text { Europe } \\
(n=168)\end{array}$ & $\begin{array}{c}\text { Australia } \\
(n=93)\end{array}$ & $\begin{array}{l}\begin{array}{l}\text { Other } \\
\text { country } \\
(n=155)\end{array} \\
\end{array}$ & $P$ value \\
\hline Shoulder & 28.9 & 28.4 & 42.1 & 29.2 & 24.7 & 31.6 & $0.011+$ \\
\hline Iliopsoas muscle & 22.9 & 10.7 & 19.7 & 7.7 & 14.0 & 21.3 & $<0.001^{*}$ \\
\hline Digits (toes) & 17.1 & 17.2 & 16.3 & 23.2 & 18.3 & 20.0 & 0.48 \\
\hline Lumbar spinal or lumbosacral & 15.8 & 20.0 & 12.4 & 17.3 & 19.4 & 27.7 & $0.003 *$ \\
\hline Stifle & 15.1 & 12.1 & 11.8 & 13.1 & 9.7 & 6.5 & 0.051 \\
\hline Thoracic spinal & 13.6 & 12.6 & 11.8 & 11.3 & 12.9 & 14.8 & 0.92 \\
\hline Paw pads & 9.5 & 12.6 & 7.9 & 11.3 & 9.7 & 14.2 & 0.31 \\
\hline Neck & 11.7 & 4.7 & 12.4 & 7.1 & 8.6 & 7.7 & $0.014 \dagger$ \\
\hline Hip & 8.4 & 14.4 & 6.2 & 3.0 & 5.4 & 12.9 & $<0.00 I^{*}$ \\
\hline Carpal & 7.5 & 10.7 & 7.9 & 11.3 & 14.0 & 7.1 & 0.12 \\
\hline Thigh & 4.7 & 8.8 & 4.5 & 6.0 & 9.7 & 10.3 & $0.011+$ \\
\hline Elbow & 4.2 & 4.7 & 7.3 & 7.1 & 2.2 & 3.2 & 0.19 \\
\hline Metacarpal & 2.9 & 2.8 & 2.8 & 4.8 & 4.3 & 4.5 & 0.59 \\
\hline Ribs or side & 2.3 & 1.4 & 3.4 & 4.2 & 3.2 & 5.8 & 0.11 \\
\hline Antebrachium & 2.0 & 1.9 & 5.6 & 0.6 & 2.2 & 0.6 & $0.033 \dagger$ \\
\hline Head & 2.0 & 0.5 & 1.7 & 2.4 & 0.0 & 5.2 & $0.042 \dagger$ \\
\hline Tibial or fibular & 2.0 & 0.5 & 1.7 & 3.6 & 0.0 & 0.6 & 0.17 \\
\hline Tarsal or calcaneal tendon & 1.5 & 2.3 & 2.2 & 2.4 & 2.2 & 1.3 & 0.74 \\
\hline Tail & 1.0 & 1.4 & 0.6 & 0.6 & 0.0 & 4.5 & $0.031 \dagger$ \\
\hline Metatarsal & 0.8 & 2.3 & 0.6 & 3.0 & 2.2 & 0.6 & $0.047 \dagger$ \\
\hline
\end{tabular}

Data represent percentage of dogs of each country or region reported to have an injury in that anatomic region.

*Significant difference among geographic regions after Holm correction for multiple comparisons among the 20 anatomic regions. †Significant difference among geographic regions without Holm correction for multiple comparisons.

significantly $(P<0.001)$ more common among dogs in the US and Canada and less common among dogs in the UK and Ireland and Continental Europe. Lumbosacral injuries were significantly $(P=0.003)$ more common among dogs in the UK and Ireland, Australia, and other countries and less common among dogs in the US and Canada. Injuries of the hip region were significantly $(P<0.001)$ more common among dogs in the UK and Ireland and less common among dogs in Continental Europe. For these 3 locations, significant variations among regions were still identified after use of the Holm correction for multiple comparisons. For other locations, including the shoulder region, neck, thigh, antebrachium, head, tail, and metatarsal region, differences among regions were significant at values of $P<0.05$.

\section{Discussion}

Results of the present study suggested that musculoskeletal injuries were common among dogs competing and training in agility, with injuries to the shoulder region substantially more common than injuries in other anatomic locations and iliopsoas muscle injuries second most common. In addition, we found that the frequency and types of injuries varied among breeds and geographic regions. The overall prevalence of injuries in the present survey was $41.7 \%(1,958 / 4,701)$, which was substantially higher than the $32 \%$ reported a decade ago. ${ }^{2}$ Importantly, most of the dogs in the present study were $\leq 11$ years old, meaning that they would not have been included in the earlier survey.

The apparent increase in the frequency of injuries in the present study coincided with substantial changes in the sport with regard to level of competition and technical challenges but also with substantial advances in the field of canine sports medicine and rehabilitation. Veterinary sports medicine and rehabilitation did not become a recognized specialty until 2010 , and since that time, the number of specialists in this area has grown, the education and knowledge of veterinarians has increased, and advanced diagnostic modalities have become more widely available. As a result, the awareness and diagnosis of sports-related injuries has likely increased over the past decade, which may have contributed to the increase in the frequency of injuries reported in the present study. Educational opportunities for the general public have also become widely available, which has increased handler awareness of injuries. However, many of the educational opportunities targeted to handlers and veterinary professionals are focused on specific injuries, which could lead to increased diagnosis and potentially overdiagnosis of those particular injuries.

Border Collie is the most common breed participating in agility and was also the most commonly injured breed in our study, which was consistent with findings of previous studies. ${ }^{1,3}$ However, the percentage of Border Collies for which an injury was reported (51.9\%) was substantially higher than previously reported (41\%). ${ }^{3}$ It is possible that the increase in competitiveness and faster course times over the past decade, coupled with the fast speed of Border Collies in general, have increased the overall risk of injury. Additional studies to determine whether a correlation between speed and injury truly exists are needed to substantiate this hypothesis.

Agility course design varies by sponsoring organization, region, venue, and judge. Differences 
in course requirements and design, along with differences in training and handling methods, could have contributed to the variation in injury frequency among geographic regions in the present study. The percentage of dogs with an injury was highest in Australia (53.5\%), and course design in Australia varies from that in much of the rest of the world. In Australia, the minimum spacing between obstacles is $4 \mathrm{~m}$ (13 feet) and the maximum spacing is $8 \mathrm{~m}$ (26 feet), ${ }^{19}$ with most courses in Australia built with the minimum distance between obstacles. In contrast, in the US, where the percentage of dogs with an injury was the lowest, the minimum distance between obstacles in trials licensed by the American Kennel Club is 4.5 $\mathrm{m}$ ( 15 feet) with a maximum spacing of $9 \mathrm{~m}$ (30 feet). However, the minimum distance between bar jumps is $5.5 \mathrm{~m}$ (18 feet) and the minimum spacing between spread jumps is $6.4 \mathrm{~m}$ (21 feet). ${ }^{13}$ The tighter spacing in Australian courses could potentially lead to a higher injury frequency because courses are more difficult to navigate, particularly for larger dogs. A study by Birch et $\mathrm{al}^{24}$ evaluating differences in dog kinematics and joint angulations when jumps were spaced 3.6, 4, and $5 \mathrm{~m}$ apart showed that there was increased neck angulation and increased shoulder joint flexion when jumps were spaced $4 \mathrm{~m}$ apart, compared with $5 \mathrm{~m}$ apart. This increased joint angulation during closer-spaced jumping could be contributing to the increased frequency of injuries in Australia.

Additionally, certain obstacles (eg, broad jump, tire jump, and spread jump) are required in every course for trials licensed by the Australian National Kennel Council, whereas these obstacles are not required on all courses for trials in other countries.,9-19 The higher frequency of these particular obstacles could also have influenced the injury frequency seen in Australia. Other factors that could have affected differences in the frequency of injuries in Australia versus other countries include variations in training practices, differences in injury prevention strategies, and differences in the availability of veterinarians trained in sports medicine and rehabilitation and advanced diagnostic practices. It is also possible that the gene pool for purebred dogs is smaller in Australia and that decreased genetic variation had an impact on the frequency of injuries.

In the present study, there were differences in the frequency and types of injuries between geographic regions. Injuries of the lumbosacral and lumbar spinal regions were more commonly reported in the UK and Ireland, Australia, and other countries and less commonly reported in the US and Canada. Studies by Birch et al have shown a significant increase in extension of the sacroiliac region at higher jump heights ${ }^{25}$ and variations in flexion and extension of the lumbar portion of the spine with different spacing between jumps. ${ }^{24}$ The A-frame obstacle is commonly used in agility courses and has been associated with a higher risk of injury. ${ }^{1-3}$ Kinematic analysis of dogs performing the A-frame showed that the range of motion in the lumbar portion of the spine varied with the phase of obstacle completion, with increased extension during the approach-to-incline and apex-to-decline phases and increased flexion during the incline-to-apex phase. ${ }^{26}$ It is currently thought that increased or altered mechanical load on the lumbosacral disk contributes to the high prevalence of lumbosacral disk degeneration. ${ }^{27}$ Therefore, variations in jump height, course spacing requirements, and use of the A-frame could be contributing to variation in the frequency of injuries to the lumbosacral and lumbar spinal region. It is also possible that variations in training methods for jump obstacles and the A-frame (eg, allowing the dog to run through the contact zone when exiting the obstacle versus requiring the dog to stop), age at which training is started, and frequency or repetition of training particular obstacles could all have an influence on the likelihood of injuries related to altered mechanical loads.

Iliopsoas muscle injuries were not evaluated in previous agility injury studies ${ }^{1-3}$ but were reported in $19.4 \%$ of dogs with an injury in the present study and were significantly more common in the US and Canada and significantly less common in the UK and Ireland and Continental Europe. To our knowledge, there is little research currently available regarding iliopsoas muscle injuries. The high number of iliopsoas muscle injuries observed in the present study indicates that more research is needed to evaluate the biomechanics of this muscle, the pathophysiology of injuries, and potential treatment and prevention strategies.

The frequency of injuries in the shoulder region was substantially higher than the frequency of injuries in other anatomic locations and was higher than the frequency previously reported. ${ }^{1,2}$ A study by Cullen et $\mathrm{al}^{28}$ described activation patterns of the biceps brachii, supraspinatus, infraspinatus, and triceps brachii muscles during the agility-specific tasks of jumping and ascending and descending the A-frame. That study showed that the jump task is the most demanding across all evaluated forelimb muscles. Another more recent study ${ }^{29}$ evaluated limb length and stiffness during jumping in beginner and advanced agility dogs. The results of the study showed that, during the stance phase of landing, beginner dogs had $17 \%$ higher limb compression, which was also associated with a greater amount of eccentric muscle contraction. Eccentric muscle contractions have been shown to contribute to injury in people. ${ }^{30}$ Thus, the high muscle activation during jumping, eccentric loading of the forelimb muscles, and high frequency of jump obstacles in agility courses could be causes of the high reported frequency of shoulder region injuries. The jump height relative to the height of the dog, experience level of the dog, experience level of the handler, training and handling methods, frequency and repetition of training, surface type, and strength and conditioning of the dog could all affect both 
jumping ability and form, thereby also influencing the frequency and types of shoulder region injuries. Currently available studies regarding jumping are not completely representative of jumping in agility training and competition because they only involve straight line jumping. To our knowledge, there have been no evaluations of muscle activation, kinematics, or ground reaction forces when dogs are jumping in collection versus extension or approaching jumps at various angles or speeds. A study by Pfau et $\mathrm{al}^{31}$ found that landing angle was significantly correlated with vertical impulse, peak vertical force, and acceleration impulse, particularly in regard to the forelimbs, and suggested that the steeper the landing angle, the potentially higher the joint moments. More difficult jump approaches and approaches to the backsides of jumps may result in steeper landing angles, thereby increasing the forces on the joints in the forelimbs and the likelihood of injury. Further biomechanical studies are needed to evaluate the effect of more difficult jump approaches on shoulder muscle activation and kinematics.

Unlike the case for other anatomic regions, the reported frequency of digit injuries was consistent with frequencies reported previously. ${ }^{1,2,32}$ There were also no observed differences in frequency of digit injuries among geographic regions in the present study. The lack of regional differences and lack of change over the past decade may indicate that variations in course design between countries and the increasing difficulty of obstacle approaches has not changed the likelihood of digit injuries, unlike injuries involving other anatomic locations. This lack of difference in digit injury frequency also suggested that differences observed in the frequency of injuries in other anatomic locations may have represented true time-trend differences and not just a difference in survey participants. However, it is also possible that digit injuries are easier to diagnose than injuries of the shoulder region, back, or iliopsoas muscles and are more easily identified by owners and thus more consistently noted.

Limitations of the present study included potential inaccuracies related to participant recall and the inclusion of owner-reported injuries that may not have been confirmed by a veterinarian. Self-selection bias may also have resulted in the sample not being representative of the total agility dog population. Injuries reported were not necessarily related directly to participation in agility, and because there are no data regarding typical injury rates in the general canine population, it is impossible to determine whether dogs competing and training in agility have a higher incidence of injury than the general population.

There was an overwhelming response to our survey by the agility community. The survey was started by 5,113 individuals and completed by 4,197 . Median response time among those who completed the survey was 18 minutes. Some individuals may not have completed the survey because of its length or because of language difficulties or differences in terminology among countries. Still, the number of responses and high completion rate were evidence of the agility community's interest in learning how to help prevent injuries. Competitors are highly invested in their dogs and are looking for ways to improve their safety and performance.

Our findings provide valuable insights into the most commonly reported injuries in agility dogs and may help guide clinical evaluations and diagnostic testing of dogs examined because of lameness or performance issues. The survey also indicated a need for studies evaluating the biomechanics of injuries to the shoulder region, iliopsoas muscle, and lumbar spinal region as well as studies aimed at prevention and treatment strategies for these injuries. The significantly higher frequency of injuries among Border Collies, compared with dogs of other breeds, requires additional evaluation, as does the variability in the frequency of injuries among geographic regions. As the popularity of companion dog sports continues to grow, there is an increasing need for research on injuries specific to individual sports so that veterinarians can provide the best care and training recommendations.

\section{Acknowledgments}

No third-party funding or support was received in connection with this study or the writing or publication of the manuscript. The authors declare that there were no conflicts of interest.

\section{References}

1. Cullen KL, Dickey JP, Bent LR, et al. Survey-based analysis of risk factors for injury among dogs participating in agility training and competition events. J Am Vet Med Assoc 2013;243(7):1019-1024.

2. Cullen KL, Dickey JP, Bent LR, et al. Internet-based survey of the nature and perceived causes of injury to dogs participating in agility training and competition events. J Am Vet Med Assoc 2013;243(7):1010-1018

3. Levy M, Hall C, Trentacosta $\mathrm{N}$, et al. A preliminary retrospective survey of injuries occurring in dogs participating in canine agility. Vet Comp Orthop Traumatol 2009;22(4):321-324.

4. Agility judges' guidelines 2010. American Kennel Club. Accessed June 24, 2020. https://images.akc.org/pdf/events/ agility/judges_guidelines_2010.pdf

5. Agility judges' guidelines 2020. American Kennel Club. Accessed June 21, 2020. https://images.akc.org/pdf/rulebooks/ REAJG1.pdf

6. AKC agility suspends use of the collapsed tunnel 2016. American Kennel Club. Accessed June 23, 2020. https://www.akc.org/ expert-advice/news/akc-agility-suspends-use-of-the-collapsedtunnel/

7. Revisions to the regulations for agility trials 2018. American Kennel Club. Accessed June 23, 2020. https://images.akc.org/pdf/ events/agility/0217_Equipment_Changes_Chapter_3_insert_ Corrected_.pdf

8. Break-away tire 2013. American Kennel Club. Accessed June 23, 2020. https://images.akc.org/pdf/events/agility/ Announcement-BreakawayTireAug2013.pdf

9. Official rules and regulations of United States Dog Agility Association Inc 2011. United States Dog Agility Association Inc. Accessed June 21, 2020. https://www.usdaa.com/regulations/ usdaa-rules-and-regulations.cfm

10. RULESIFCS Agility Competitions 2017. International Federation of Cynological Sports. Accessed June 21, 2020. https:// ifcsdogsports.org/wp-content/uploads/2017/04/IFCS-AgilityRules-Dated-February-2017.pdf

11. FCI agility regulations 2018. Federation Cynologique Internationale (AISBL). Accessed June 21, 2020. www.fci.be/en/ Agility-45.html 
12. Agility regulations 2019. Agility Dog Association of Australia Ltd. Accessed June 21, 2020. https://adaa.com.au/info/wpcontent/uploads/adaa-regs-e10-1.pdf

13. Regulations for agility trials and Agility Course Test 2020. American Kennel Club. Accessed June 21, 2020. https://images.akc. org/pdf/rulebooks/REAGIL.pdf

14. AKC agility trial manual 2020. American Kennel Club. Accessed June 21, 2020. https://images.akc.org/pdf/agility_trial_manual. pdf

15. Amendments to official rules \& regulations 2020. United States Dog Agility Association Inc. Accessed June 21, 2020. https:// www.usdaa.com/regulations/UpdatesRulesRegulations.cfm

16. Exhibitor's handbook for guidelines governing North American Dog Agility Council sanctioned trials 2020. The North American Dog Agility Council. Accessed June 21, 2020. https://www.nadac. com/WPsite/wp-content/uploads/2020/04/NADAC-Exhibitor_ MasterFile-Rev-2.25.20.pdf

17. Rules and regulations sixth edition 2020. UK Agility International. Accessed June 21, 2020. https://ukagilityinternational. com/wp-content/uploads/2020/06/UKI-6th-Edition-RulesMay-2020-6.11.pdf

18. 2020 rulebook. Canine Performance Events. Accessed June 21, 2020. https://www.k9cpe.com/forms/2020rb.pdf

19. Rules for the conduct of agility trials 2018. Australian Nation al Kennel Council Ltd. Accessed June 24, 2020. https://ankc. org.au/media/9061/1-agilityrules-2018.pdf

20. Hillebrand L. 2009 FCI Agility World Championship results. Accessed June 25, 2020. https://www.lhbsystems.nl/ wc2009/default.asp

21. 2018 FCI Agility World Championship results. SvenskaAgilityklubben. Accessed June 25, 2020. https://sagikvmfrontend.
azurewebsites.net/?fbclid=IwAR1sNZpINIdEc8DcJpzoRaL6Cj bkW4yMjschAvQxMY_Smj689dXXNVTbgTM

22. Anderson TM, McIlwraith CW, Douay P. The role of conformation in musculoskeletal problems in the racing Thoroughbred. Equine Vet J. 2004;36(7):571-575.

23. Mostafa MB, Elemmawy YM. Relationships between morphometric measurements and musculoskeletal disorders in jumping Thoroughbred horses. J Equine Sci. 2020;31(2):23-27.

24. Birch E, Boyd J, Doyle G, et al. The effects of altered distances between obstacles on the jump kinematics and apparent joint angulations of large agility dogs. Vet J. 2015;204(2):174-178.

25. Birch E, Leśniak K. Effect of fence height on joint angles of agility dogs. Vet J. 2013;198(suppl 1):e99-e102.

26. Williams JM, Jackson R, Phillips C. Kinematic analysis of the A-frame in canine agility. J Small Anim Pract. 2016;57(supp1 2):5. Abstract

27. Meij BP, Bergknut N. Degenerative lumbosacral stenosis in dogs. Vet Clin North Am Small Anim Pract. 2010;40(5):983-1009.

28. Cullen KL, Dickey JP, Brown SH, et al. The magnitude of muscular activation of four canine forelimb muscles in dogs performing two agility-specific tasks. BMC Vet Res. 2017;13(1):68.

29. Söhnel K, Rode C, de Lussanet MHE, et al. Limb dynamics in agility jumps of beginner and advanced dogs. J Exp Biol. 2020;223(pt 7):jeb202119.

30. Dueweke JJ, Awan TM, Mendias CL. Regeneration of skeletal muscle after eccentric injury. J Sport Rehabil. 2017;26(2):171-179.

31. Pfau T, Garland de Rivaz A, Brighton S, et al. Kinetics of jump landing in agility dogs. Vet J. 2011;190(2):278-283.

32. Sellon DC, Martucci K, Wenz JR, et al. A survey of risk factors for digit injuries among dogs training and competing in agility events. J Am Vet Med Assoc. 2018;252(1):75-83. 